\title{
LA TUTORÍA AFECTIVA Y EL CAMBIO TERAPEÚtICO
}

\author{
Cristina Santana Navarro \\ Psicóloga. Máster en Formación del Profesorado \\ Ma Elena Díaz-Negrín \\ Consejería de Educación Gobierno de Canarias \\ Isabel Luján Henríquez \\ Departamento de Psicología, Sociología y Trabajo Social. \\ Universidad de Las Palmas de Gran Canaria. \\ isabel.lujan@ulpgc.es
}

Fecha de Recepción: 7 Abril 2018

Fecha de Admisión: 10 Abril 2018

\section{RESUMEN}

El presente trabajo recoge el diseño de un programa de intervención individualizada mediante tutorías afectivas, cuyo objetivo es generar cambios tanto en la manera de afrontar la realidad la población adolescente como de formar a tutores afectivos.

La propuesta surge, por un lado, desde la reflexión realizada sobre la gran cantidad de jóvenes que manifiestan conductas desadaptadas debido a conflictos en diversos ámbitos de su vida y cuya influencia y /o consecuencias se suelen observar en los centros escolares. Por otro lado, desde la apreciación de falta de claridad y de sistematización existente en los diseños y programas relativos a las tutorías afectivas. Así, hemos encontrado un vacío sobre cuáles podrían ser las medidas reeducadoras para aplicar en cada caso, pues no se han definido instrumentos concretos que pueda emplear el tutor-afectivo, ni delimitado de forma clara y concisa la población participante, ya que estas tutorías se llevan a cabo sin designar a un/a profesional formado en habilidades y conocimientos para servir de ayuda y apoyo desde la atención a la diversidad.

En consecuencia, se diseña una propuesta de intervención individualizada con la pretensión de superar esta falta de concreción y la ambigüedad en relación a qué son las tutorías afectivas, qué variables van asociadas a las mismas y cómo deber ser el proceso de tutorización.

Es una propuesta fundamentada en modelos teóricos concretos (teoría de los moldes cognitivo-afectivos de Hernández-Guanir y terapias contextuales de tercera generación), en la que se delimita criterios claros para seleccionar a los sujetos participantes, se diseñan instrumentos y materiales específicos y se establecen sesiones formativas para los tutores afectivos.

Los resultados aportados a través de entrevistas en profundidad, realizadas a una muestra piloto de docentes que han implementado el programa, señalan como apropiado el procedimiento esta- 


\section{LA TUTORÍA AFECTIVA Y EL CAMBIO TERAPEÚTICO}

blecido, pues consideran que atiende al alumnado en su dimensión personal, afectiva y social con resultados positivos en todas las áreas.

Palabras clave: tutoría afectiva; conducta desadaptada; cambio terapéutico; atención a la diversidad; moldes cognitivo-afectivos

\section{ABSTRACT}

\section{The affective tutorship and the therapeutic change.}

The present work consists in the design of a proposal of an individualized intervention program, through affective tutoring, whose main objective is to generate possible changes in the way of facing the reality in the adolescent population, besides training the affective tutors who support and guide young people in their process of change.

The proposal arises from the reflection carried out, on the one hand, by the number of cases of young people who exhibit maladaptive behaviours. This maladaptation or maladjustment causes conflicts in different areas of life whose influence and / or consequences are frequently observed in schools. On the other hand, from the appreciation of the lack of clarity and systematization existing in a resource offered as an element of help and support from the attention to diversity: affective mentoring. Thus, we have found a gap on what reeducation measures could be applied in each case, since no specific instruments have been defined that can be used by the affective tutor, nor clearly and concisely delimited by the participating population, since these tutorials are carry out without designating a professional trained in skills and knowledge to serve as help and support from the attention to diversity.

Consequently, a proposal for individualized intervention is designed with the aim of overcoming this lack of concreteness and ambiguity in relation to what affective tutoring is, what variables are associated with them and how the tutoring process should be.

It is a proposal based on specific theoretical models (theory of the cognitive- affective molds of Hernández-Guanir and third-generation contextual therapies), in which clear criteria are defined to select the participants, specific instruments and materials are designed and They establish sessions that offer specific training for the affective tutors as well as in the techniques and skills necessary for the intervention with the student.

The results provided through in-depth interviews conducted with a pilot sample of teachers who have implemented the program, indicate as appropriate the procedure established, since they consider that it attends to the students in their personal, affective and social with positive results in all areas.

Keywords: affective mentoring; maladaptive behaviour; therapeutic change; attention to diversity; cognitive-affective moulds

\section{ANTECEDENTES}

Las personas necesitamos dar sentido al mundo social que nos rodea y manejar una ingente cantidad de información para conseguir una adecuada adaptación. Pero la mente humana tiene una capacidad limitada para procesar esa cantidad de información, por lo que escatimamos esfuerzos y buscamos atajos para simplificar todo lo posible las cosas y encontrar soluciones rápidas que nos permitan actuar de forma eficaz. Esto lo hacemos recurriendo a diferentes estrategias mentales, que, sin ser siempre conscientes de ellas (Gaviria, Cuadrado y López, 2013), y que aunque a veces se produzcan errores y distorsiones, nos permiten comprender el mundo y la vida así como afrontar la realidad de forma favorable y satisfactoria.

También es sabido que las emociones son las herramientas fundamentales de adaptación, ya que se asocian a formas de pensamiento idóneas para afrontar situaciones de cotidianeidad 
(Hernández-Guanir, 2010). Las emociones son el resultado evaluativo de un proceso cognitivo. Existen unos procesadores cognitivos que hacen disparar las respuestas emocionales para regular el comportamiento (Laird, 2000). La interpretación cognitiva de los sucesos es gestionada por elementos simbólicos, que activan las estrategias socio-afectivas (Rodríguez-Mateo, Díaz-Negrín, Torrecillas, Luján y Rodríguez-Rodríguez, 2016), Ilamadas moldes mentales por el profesor Hernández- Guanir $(2002,2010)$. Estas variables actúan como mediadoras entre lo emocional y lo conductual y permiten una adaptación 0 ajuste inteligente.

El ajuste inteligentemente adaptativo ha sido abordado desde distintas teorías. Así, es posible encontrar, la perspectiva personal que se centra en variables personales como explicativas de la visión del mundo de los sujetos. En esta línea destacan las teorías atribucionales de Weiner (1986), la teoría de Kelly $(1955)$, la teoría de Ellis $(1957,1977)$ o la teoría de Wessler (1993) como líneas relacionadas con la propuesta de los Moldes cognitivo-afectivos, Hernández-Guanir (2002, 2010).

Existen otras perspectivas que defienden la valoración de la realidad como acto inteligente. Uno de estos enfoques es el de las denominadas, Inteligencias socioafectivas. Éstas entienden la realidad de los sujetos en base a su inteligencia, pero no sólo como producto, sino como elemento de eficacia. Destacan aquí, Sternberg (1985, 1997), Gardner (2008), Mayer, Salovey y Caruso (1999, 2008) o Csikszentmihalyi (2003). Estas teorías tienen en común el concebir la adaptación como un proceso inteligente que requiere de habilidades intelectuales que se desarrollan, por un lado, gracias a la carga genética y por otro, a través del desarrollo de la propia interacción con el medio y la experiencia en éste. Pero para todo ello, hay que añadir a esas habilidades intelectuales, unas habilidades personales. Se habla entonces, de una inteligencia que contribuye a que las personas se encuentren adaptadas de una forma óptima y eficaz a sí mismos, a su entorno y a su realidad.

Finalmente, existen teorías que aportan un aspecto más concreto y operativo. Proponen respuestas cognitivo-afectivas que operativizan la evaluación de la realidad mediante habilidades inteligentes, y ofrecen estrategias concretas para explicar la conducta de eficacia y de valoración de la realidad como acto inteligente. En esta perspectiva se enmarcan planteamientos como los de Lazarus (1986), Lazarus y Folkman (1993), Beck (1976) o Mischel $(1968,1981)$. En esta perspectiva se encuadra la teoría de autorregulación cognitivo-afectiva de Hernández-Guanir $(2002,2010)$ de los moldes como estrategias específicas y modificables.

Desde la perspectiva de los moldes mentales, estos se presentan como las unidades básicas de análisis dentro de lo que Hernández-Guanir $(2002,2010)$ sugiere como inteligencia para la vivencia. Suponen estrategias cognitivas autorreguladoras, que constituyen una base realista y operativa para la construcción de un modelo de inteligencia esencialmente cognitiva, estratégica e integradora, de lo emocional, lo cognitivo y lo conductual.

Para Hernández-Guanir, lo relevante son las estrategias que tenga la mente para ayudarnos a conocernos y conducirnos mejor. A esta capacidad de conocimiento y conducción la denomina inteligencia o potencial socioafectivo. Se consideran estrategias cognitivas que permiten conocer y manejar las propias cogniciones, emociones y conductas, pero se enfatiza en los procesos mentales que intervienen en el ajuste de las personas al ambiente.

A partir de ello se defiende la existencia de los denominados moldes cognitivo- afectivos. Suponen la manera de construir la realidad que nos rodea y en la que nos desenvolvemos. Éstos se definen como estrategias particulares y habituales que cada persona muestra en el modo de interpretar y reaccionar ante la realidad en situaciones de egoimplicación. Estas situaciones son aquellas en las que las personas se enfrentan a una realidad que les compromete en sus intereses y emociones.

Se destaca de los moldes mentales que se trata de constructos que definen la manera en que 


\section{LA TUTORÍA AFECTIVA Y EL CAMBIO TERAPEÚTICO}

describen y/o afrontan una realidad las personas. No es tan importante el contenido de los moldes, sino la forma en que se "tratan" estos contenidos mentales.

Según esta teoría, la mente se vale de estrategias para interpretar la realidad y reaccionar ante ella, y con el paso del tiempo y su puesta en práctica ante diferentes situaciones y exigencias ambientales, estas estrategias van consolidando unos patrones mentales que Hernández-Guanir denomina "hábitos mentales de actuación". De esta manera, mediante el empleo repetido de unos mismos "hábitos mentales" en diversas ocasiones y situaciones se fomenta que, en futuras actuaciones realicemos las mismas operaciones mentales. Así resulta muy probable que una persona genere patrones mentales estables, 0 incluso crónicos, puesto que los moldes que tendemos a emplear se van automatizando con su práctica, el paso del tiempo y la generalización en diversas situaciones.

Desde esta perspectiva se plantea una Inteligencia socioafectiva que implique el conocimiento y gobierno de las cogniciones, las emociones y las conductas, tanto propias como ajenas. Una adecuada inteligencia socioafectiva nos permitirá manejar el sufrimiento personal y el modo de desenvolvernos en la vida, para manejarnos a nosotros mismos o a la realidad social que nos envuelve.

A partir de planteamientos como los expuestos Hernández-Guanir $(2002,2010)$ desarrolla el enfoque de las Estrategias socioafectivas. Defiende la capacidad de distinguir e interpretar nuestros pensamientos, sentimientos o comportamientos, relacionándolos y encontrando soluciones en nuestra vida. Por ello, es posible decir que la inteligencia para la vivencia, tal como la llama, es a su vez, una inteligencia al servicio de la eficiencia. Dicha teoría es la que fundamenta el programa de intervención individualizada: PITAC, La tutoría afectiva como herramienta para el cambio, (Santana, Rodríguez-Mateo y Luján, 2017).

Hernández-Guanir indica que las estrategias cognitivas autorreguladoras, constituyen una base realista y operativa para la construcción de un modelo de inteligencia esencialmente cognitiva, estratégica e integradora, de lo emocional, lo cognitivo y lo conductual. Son estrategias que facilitan una adaptación 0 ajuste inteligente, y a su vez, actúan como mediadoras entre lo emocional y lo conductual.

Ahora bien, todo proceso adaptativo conlleva un cambio, ya que se modifica y se transforma la manera de enfrentarnos a nuestra realidad. Este proceso de cambio ha sido interpretado a lo largo de la historia por diferentes teorías hasta llegar a lo que se denominan teorías de última generación, y dentro de ellas las terapias de tercera generación (Linehan 1993; Teasdale, Moore, R. G., Hayhurst, H., Pope, M., Williams, S., \&Segal, Z. V, 2002), que ponen el énfasis en el análisis de la conducta de la persona (producto de sus evaluaciones cognitivas sobre sí mismo o las situaciones), pero destacando de ella la conducta verbal y la clarificación de valores.

Estas teorías defienden que el cambio supone no continuar evaluando las situaciones problemáticas, sino en buscar alternativas eficaces y orientarse en una solución basada en aquello que sea prioritario, relevante y valioso en la vida. Defienden que se debe buscar el cambio reorientando la vida de la persona, sin importar si dichos problemas se reducen o no, y para ello se centran en modificaciones en el lenguaje interno de los sujetos y en su conducta. Esto genera, que las evaluaciones y lenguaje interno, al ser contrastados con sus acciones en los diferentes contextos, que antes se evitaban, se modifiquen también.

En base a todo ello, y sabiendo que cambiar es conseguir una verdadera modificación que suponga transformaciones en la manera de enfrentarnos a nuestra realidad, diseñamos un programa de intervención individualizada (PITAC) que pretende generar posibles cambios terapéuticos en los individuos, y transferir estos a todos los ámbitos de la vida de la persona. 


\section{OBJETIVOS DE LA INVESTIGACIÓN}

El objetivo fundamental del programa de intervención individualizada a través de tutorías afectivas, es conseguir generar posibles cambios en la manera de afrontar la realidad que viven los jóvenes, así como en sus actitudes y comportamientos. El otro objetivo clave del mismo es dar formación específica a docentes con la finalidad de intentar conseguir, mediante el desarrollo de las sesiones de tutoría, generar tales cambios en los jóvenes receptores.

Esta propuesta surge al observar cómo en diversos ámbitos existen jóvenes con dificultades a la hora de desenvolverse en su día a día sin que ello sea consecuencia de dificultades de aprendizaje, ni de problemas cognitivos. Sin embargo, estos conflictos desencadenan problemáticas diversas en sus vidas, que se aprecian a través de actitudes y acciones que incitan a faltas de respeto, discusiones, incidencias reiteradas, comportamientos inadecuados, actitudes desafiantes, conductas de riesgo etc. en diferentes contextos. Todo esto suele influir en su rendimiento escolar, en el absentismo escolar, en la relación con docentes y compañeros, en la realización de tareas, en el seguimiento y participación de las dinámicas en el aula, etc.

A pesar de la problemática que se genera, el sistema educativo no les suele ofrecer recursos efectivos para tratar tales dificultades. En muchas ocasiones, se les deriva a recursos que no resultan ser de ayuda en su problemática, dentro de los cuales está la tutoría afectiva.

Tras apreciar todo ello desde la propia experiencia profesional y el análisis de los protocolos existentes, es posible afirmar que la tutoría afectiva, tal como se está llevando a cabo actualmente, resulta ineficaz o bastante menos beneficiosa de lo que se espera, pues se presenta sin un diseño sistematizado que organice y guíe las sesiones y, además, no existe formación para los docentes que sean tutores afectivos. Por tanto, es posible encontrar profesionales con formación académica muy diversa, que, en muchas ocasiones, desconocen completamente los contenidos, técnicas 0 habilidades necesarias para llevarlas a cabo. Tampoco se dispone de instrumentos que sirvan para identificar las causas que están generando y/o incidiendo en la problemática que manifiestan los jóvenes. Menos aún, existen materiales y recursos específicos que sirvan de apoyo al tutor afectivo a la hora de llevar a cabo las sesiones de tutoría.

Por todo ello, se plantea un programa de intervención individualizada -PITAC- que pretende dar respuesta a esas lagunas en cuanto a claridad, concreción y sistematización a la hora de llevar a cabo estas tutorías. Se contemplan por ello, por un lado, objetivos para docentes y por otro, objetivos para trabajar con el alumnado.

\section{Objetivos para docentes:}

Formar en dimensiones focales y estrategias socioafectivas, mediante la Teoría de los moldes cognitivo-afectivos de Hernández-Guanir, (2002, 2010).

Formar en la entrevista individual semiestructurada sobre estrategias cognitivo- afectivas y su uso, para detectar patrones de pensamiento inadecuados.

Formar en técnicas y habilidades de comunicación verbal y no verbal, para el desarrollo de la entrevista e interacción con los jóvenes durante las sesiones.

Dar a conocer recursos, material y actividades que se desarrollarán en las sesiones, según cada caso.

\section{Objetivos dirigidos al alumnado:}

Capacitar a los jóvenes para identificar estrategias socioafectivas no adecuadas que están empleando.

Generar en los jóvenes la aceptación sobre la manera inadecuada de enfocar su realidad para promover, con ello, el cambio hacia otras estrategias o maneras de enfocarla. 


\section{LA TUTORÍA AFECTIVA Y EL CAMBIO TERAPEÚTICO}

Educar a los jóvenes en estrategias cognitivo-afectivas adecuadas de ajuste al medio y optimización.

Fomentar el empleo de estrategias cognitivo-afectivas de ajuste y optimización de manera que se consiga la adherencia al cambio.

Transferir el cambio hacia estrategias cognitivo-afectivas adecuadas mediante sesiones de seguimiento.

\section{MÉTODO E INSTRUMENTOS UTILIZADOS}

Para el diseño del programa de intervención individualizada, se ha empleado el método hipotético-deductivo. La metodología es descriptiva y cualitativa, y el diseño es secuencial longitudinal ya que en una aplicación práctica del programa se estudiaría su eficacia durante varios cursos, y se contrastarían los resultados a través de una evaluación pre y post a la implementación del mismo.

\section{INSTRUMENTOS}

Para el diseño y la implementación de la propuesta de programa PITAC

(Santana, Rodríguez-Mateo y Luján, 2017) se emplean los siguientes instrumentos:

Test de estrategias cognitivo -emocionales (MOLDES). Recoge las formas peculiares o formatos de pensamiento de las personas denominados Moldes Mentales o Moldes Cognitivo -afectivos (Hernández Guanir, 2002, 2010), cuyo objetivo es conocer las estrategias cognitivo-emocionales ("moldes mentales") que, normalmente, se utilizan a la hora de anticipar, interpretar, reaccionar, evaluar y explicar la realidad en aquellas situaciones que estamos envueltos o afectan a nuestra identidad, intereses o proyectos. El conocimiento de los moldes mentales facilita la comprensión, manejo y modificación de nuestras emociones y comportamientos, aspectos centrales de las inteligencias socioafectivas.

Se trata de una prueba de papel y lápiz, que consta de 87 ítems, que describen diferentes situaciones de la vida, con 5 opciones de respuesta para indicar el grado de acuerdo. Este grado varía desde 1 a 5 , donde: 1 es totalmente en desacuerdo y 5 completamente de acuerdo.

Los resultados del test se articulan de acuerdo con una estructura jerárquica formada por tres niveles: $1^{\circ}$ ) "tres encuadres focales" o factores de tercer orden; $2^{\circ}$ ) "diez dimensiones focales" o factores de segundo orden y $3^{\circ}$ ) "treinta moldes" o factores de primer orden.

El índice de consistencia interna es 0,91 alfa de Cronbach, tomando en consideración el cuestionario en su conjunto, lo que indica que cada uno de los elementos que componen el cuestionario tiene que ver con las estrategias cognitivo- emocionales. La fiabilidad de las diez dimensiones focales oscila entre el 0,75 y el 0,97 alfa de Cronbach.

Entrevista individual semiestructurada sobre estrategias cognitivo-afectivas (Rodríguez, Santana, 2017), suscitadora de intercambio conversacional para evaluar "estrategias cognitivo-afectivas", agrupadas en diez "dimensiones" que presenta el alumnado. Junto a Hoja de registro de observación e inferencia sobre las dimensiones focales.

Cuestionario de evaluación del programa intervención individualizada: La Tutoría afectiva para el cambio o PITAC.

Programa de intervención: La Tutoría afectiva como herramienta para el cambio o PITAC (Santana, Rodríguez-Mateo y Luján, 2017).

Programa de formación para tutores afectivos PITAC.

\section{POBLACIÓN}

El diseño del programa de intervención se elabora dirigido hacia dos sectores, que se conformarán como la población diana del PITAC: Los docentes como "agentes del cambio" (tutores afec- 
tivos), y los alumnos y alumnas que acudan a las tutorías afectivas como los "agentes receptores para el cambio".

\section{PROCEDIMIENTO}

Se diseña un programa de intervención individualizada con tres partes, conformadas por una serie de fases:

- Primera parte: Preparación agente del cambio.

Se lleva a cabo con docentes que se presenten voluntariamente, que estén interesados en formarse y en ser tutor/a afectivo/a. Estos pasarán por diferentes fases las cuales constituyen el Programa de formación para tutores afectivos PITAC:

Fase formación: Inicio formación grupal en la adquisición de competencias y habilidades para el desarrollo de las tutorías afectivas. Tal fase se desarrolla a través de tres bloques temáticos de contenidos, mediante sesiones formativas que oscilan de entre 1 hora y media a 2 horas. Los bloques formativos son los siguientes:

Bloque I: Los Moldes de la Mente: Dimensiones focales y moldes cognitivo-afectivos.

Bloque II: Habilidades y técnicas para el desarrollo de la entrevista e interacción durante las sesiones.

Bloque III: Empleo de la entrevista individual semiestructurada sobre estrategias cognitivo-afectivas).

Fase pre-tutorías afectivas: Se aplica el Test MOLDES, Hernández-Guanir (2010), al alumnado de las diferentes tutorías y, además, los tutores deben cumplimentar el Cuestionario de evaluación del PITAC. Asimismo, se determinarán cuáles son los/as jóvenes que se propondrán como receptores de las sesiones de tutoría afectiva.

-Segunda parte: Desarrollo sesiones Tutoría afectiva para el cambio.

Comienzo de sesiones individualizadas con cada alumno, llevadas a cabo por los tutores afectivos. Se diseñan unas fases concretas y secuenciadas para facilitar el proceso de cambio en los jóvenes que acudan a tutoría afectiva. Dichas fases, así como las tareas y recursos empleados están fundamentadas en teorías específicas con validez y fiabilidad demostrada tales como las Teoría de los moldes cognitivo-afectivos las Terapias de tercera generación. Mediante la secuencia establecida se infiere que se darán cambios a corto y medio plazo en los jóvenes.

El número de sesiones varía según cada caso concreto, siendo de aproximadamente 1 hora. Éstas se desarrollarán en cuatro fases que guiarán el proceso de cambio:

Identificación estrategias socioafectivas inadecuadas.

Distanciamiento de estrategias socioafectivas inadecuadas.

Conocimiento y empleo moldes adecuados.

Fase de seguimiento del cambio.

-Tercera parte: Post-sesiones tutoría.

Se aplicará nuevamente, el Cuestionario de evaluación del PITAC y el Test MOLDES (HernándezGuanir, 2010) en los diferentes grupos. Esta segunda aplicación se comparará con la primera aplicación de dichos instrumentos para observar y analizar la eficacia del programa. De esta forma se establecerá medida test-retest del programa de intervención individualizada.

\section{RESULTADOS}

Los resultados obtenidos son de tipo cualitativo, basados en aportaciones, a través de entrevistas en profundidad, realizadas a diferentes profesionales que han conocido y llevado a cabo el programa en una práctica-piloto del mismo. Dichos profesionales informan, a nivel general, que: 


\section{LA TUTORÍA AFECTIVA Y EL CAMBIO TERAPEÚTICO}

La implementación de un programa como el PITAC es necesaria, ya que es una herramienta que reporta beneficios directamente en la población juvenil, cuyas problemáticas, constituyen parte del reflejo de la comunidad en la que vivimos, e indirectamente en la realidad social de los centros educativos y en la propia sociedad.

Plasman una valoración positiva en relación a la perspectiva integral y holística del programa, pues atiende al alumno en su dimensión personal, afectiva y social.

Manifiestan satisfacción personal con la aplicación del programa por la mejor relación que se establece con el alumnado, ya que se genera un sentimiento personal positivo al conseguir ser una figura cercana y un referente para los jóvenes que participaron.

Perciben mayor eficacia a la hora de realizar derivaciones del alumnado que se postula para ser receptor de tutoría afectiva, pues el programa aporta criterios claros de actuación.

Aprecian mayor sensación de eficacia y capacitación al afrontar las tareas como tutor afectivo al conocer técnicas, habilidades y desarrollar competencias destinadas a tal fin, así como disponer de instrumentos y recursos que facilitan y orientan dicha tarea.

Valoran el carácter preventivo del programa ya que reduce el comportamiento problemático del alumnado y aumenta su rendimiento escolar, así como mejora la convivencia del centro educativo. Observan que genera efectos directos beneficiosos sobre la convivencia y clima escolar.

Por su parte, los jóvenes participantes en la prueba-piloto muestran una actitud colaboradora y positiva tras las iniciales dudas o reticencias; se muestran cercanos con los docentes implicados tras las primeras sesiones, según refieren ambas partes. Incluso, alguno demanda la posibilidad de acudir a sesiones de tutoría afectiva más tiempo del establecido, por lo general, semanalmente.

\section{CONCLUSIONES}

Se infiere que se ha realizado un diseño adecuado que comprende elementos de los que, hasta ahora, se carecía en relación a la tutoría afectiva y las variables asociadas a esta. Esto es así, ya que:

Se ofrece fundamentación epistemológica que fundamenta la propuesta de programa de intervención presentada.

Se establecen criterios claros sobre la población receptora del cambio (jóvenes desajustados consigo mismos, sus relaciones y su realidad). También se establecen determinadas características que se suponen en la población agente del cambio (tutores afectivos).

Se diseña un período de formación concreto fundamentado en una teoría específica con validez y fiabilidad demostrada (Teoría de los moldes cognitivo-afectivos de Hernández-Guanir (2002, 2010). Se plantean diferentes fases secuenciadas, que permitan adquirir a los docentes, las competencias y habilidades necesarias para desarrollar las sesiones de tutoría afectiva y generar posibles cambios en los jóvenes.

Se establece un instrumento concreto para la evaluación de estrategias cognitivo- afectivas mediante la entrevista con los jóvenes (Entrevista individual semiestructurada sobre estrategias cognitivo-afectivas), la cual ha demostrado ser efectiva en experiencias piloto con la misma.

Se establece una evaluación sistematizada del programa de intervención, mediante medidas test-retets para comprobar su eficacia.

A pesar de las ventajas descritas es preciso ser cautos, pues se requiere una mayor implementación del programa presentado que permita verificar la validez y excelencia del mismo. Se ve necesario llevar a cabo una evaluación sistematizada y continuada en la que quede reflejada mayor número de opiniones, así como el grado de satisfacción y los logros conseguidos o no con este programa. Esto facilitaría el poder realizar un análisis cualitativo y cuantitativo del PITAC que nos indiquen la validez, fiabilidad y eficiencia del programa y, así, modificar aquellos aspectos que requieran de 
posibles mejoras 0 ajustes para conseguir -a la postre- generar beneficios en la realidad social que se vive en los centros educativos hoy día.

\section{REFERENCIAS BIBLIOGRÁFICAS}

Beck. A. (1976). Cognitive therapy of anxiety disosders. Oxford. Csikszentmihalyi. M (2003). Fluir (Flow): Una psicología de la felicidad. Kairós. Ellis, A. (1957). Rational psychotherapy and individual psychology. Journal of

Individual Psychology. Recuperado de https://search.proquest.com/openview /3daf7b0bbaf357bfc538a15d736f25b3/1?pq- origsite=gscholar \&cbl=1816607

Ellis, A. (1977). Terapia Racional Emotiva. Mexico: Pax

Gaviria, E., Cuadrado, I. y López, M. (2013).Introducción a la Psicología Social (2ª ed.). Madrid: Sanz y Torres.

Gardner, H (2008). Inteligencias múltiples. La teoría en la práctica. Barcelona: Paidós. HernándezGuanir. P (2010). Test Estrategias cognitivo-emocionales

\section{MOLDES}

Manual. TEA Ediciones.

Hernández, P. "Guanir" (2002). Moldes de la mente: más allá de la inteligencia emocional. Tenerife y Madrid, España: Tafor y Narcea.

Kelly, A. (1955). The Psychology of Personal Constructs. Recuperado en http://www.ub.edu/perso$\mathrm{nal} / \mathrm{pcp} / \mathrm{pcp} 1 . \mathrm{htm}$

Laird. J (2000). How to eliminate illusions in reasoring. Psychonomic society.

Recuperado de https://link.springer.com/article/10.3758\%2FBF03209353?LI=true Lazarus, R. \& Folkman, S. (1993). Coping Theory and Research: Past, Present.

Recuperado en https://www.ncbi.nlm.nih.gov/pubmed/8346332

Lazarus, R y FolKman, . (1986). Estrés y procesos cognitivos. Barcelona: Martínez Roca.

Linehan. M (1993). Cognitive-behavioral Treatment of Borderline Personality Disorder. Recuperado de https://books.google.es/books?hl=es\&lr=\&id=UZim30APwe8C\&oi=fnd\&pg=PA3 $\& d q=L i n e h a n .+M+(1993) .+$ Cognitive -behavioral + Treatment +of + Borderline + Personalit y + D is order. \& o t s =q C P w 3 vW s B 8 \& sig=VtQm5wFvJGdac2XtbEXirzsl1ko\#v=onepage\&q=Linehan.\%20M\%20(1993).

$\% 20$ Cognitive- behavioral\%20Treatment $\% 20$ of $\% 20$ Borderline $\% 20$ Personality $\% 20$ Disorder.\&f=fals e

Mayer, J. D., Caruso, D. \& Salovey, P. (1999). Emotional intelligence meets traditional standards for an intelligence. Paidós.

Mayer, J. D., Salovey, P., \& Caruso, D. R. (2008). Emotional intelligence: New ability or eclectic traits? American Psychologist. Recuperado de http://psycnet.apa.org/doiLanding? doi $=10.1037 \% 2 F 0003-066 X .63 .6 .503$

Mischel, W. (1968). Personalidad y evaluación. México: Trillas. Mischel, W. (1981). Teorías de la personalidad. México: Interamericana.

Rodríguez-Mateo, Díaz-Negrín, Torrecillas, Luján y Rodríguez-Rodríguez, (2016). Variables socioemocionales y bienestar psicológico en personas mayores. International Journal of Developmental and Educational Psychology. INFAD, pp.21-37.

Sternberg, R. J. (1985). Beyond IQ: A Triarchic Theory of Intelligence. Cambridge. Recuperado de https://books.google.es/books?hl=es\&lr=\&id=jmM7AAAAIAAJ\&oi=fnd\&pg=PR1 $1 \& d q=$ sternberg $+1985 \&$ ots $=$ artAacAyrl\&sig=TGCRtdJ5j_MY9EJ8vIMJFt1MMIs\# $\mathrm{v}=$ onepage $\& \mathrm{q}=$ sternberg $1985 \& \mathrm{f}=$ false

Sternberg, R. J. (1997). The concept of intelligence and its role in lifelong learning and 


\section{LA TUTORÍA AFECTIVA Y EL CAMBIO TERAPEÚTICO}

success.American Psychologist. Recuperado en http://psycnet.apa.org/record/1997- 30052-

Teasdale, J. D., Moore, R. G., Hayhurst, H., Pope, M., Williams, S., \&Segal, Z. V. (2002). Metacognitive awareness and prevention of relapse in depression: Empirical evidence. Journal of Consulting and Clinical Psychology. Recuperado de http://psycnet.apa.org/fulltext/200202267-002.html

Weiner, B. (1986). An Atribucional Theory of Motivation and Emotion. New York: Springer-Verlag Wessler, R (1993). Enfoque cognitivos para los trastornos de personalidad. Psicología Conductual, 1, 35-50. 